\title{
Integrated Simulation Design Challenges to Support TPS Repair Operations
}

Leslie J. Quiocho, NASA Johnson Space Center, 2101 NASA Parkway, Houston TX 77058, Mail Code ER7, Phone: 281-483-8633, Email: leslie.j.quiocho@nasa.gov

Edwin Z. Crues, NASA Johnson Space Center, 2101 NASA Parkway, Houston TX 77058, Mail Code ER7, Phone: 281-483-2902, Email: edwin.z.crues@nasa.gov

An Huynh, Lockheed Martin Corporation, 2400 NASA Parkway, Houston TX 77058, Mail Code C07, Phone: 281-483-2067, Email: an.huynh1@jsc.nasa.gov

Hung T. Nguyen, Lockheed Martin Corporation, 2400 NASA Parkway, Houston TX 77.058, Mail Code C07, Phone: 281-483-8180, Email: hung.t.nguyen1@jsc.nasa.gov

John MacLean, METECS, 15810 Parksley, Houston TX 77059, Phone: 832-476-8651, Email: imaclean@metecs.com

\begin{abstract}
During the Orbiter Repair Maneuver (ORM) operations planned for Return to Flight (RTF), the Shuttle Remote Manipulator System (SRMS) must grapple the International Space Station (ISS), undock the Orbiter, maneuver it through a long duration trajectory, and orient it to an EVA crewman poised at the end of the Space Station Remote Manipulator System (SSRMS) to facilitate the repair of the Thermal Protection System (TPS): Once repair has been completed and confirmed, then the SRMS proceeds back through the trajectory to dock the Orbiter to the Orbiter Docking System. In order to support analysis of the complex dynamic interactions of the integrated system formed by the Orbiter, ISS, SRMS, and SSRMS during the ORM, simulation tools used for previous 'nominal' mission support required substantial enhancements. These upgrades were necessary to provide analysts with the capabilities needed to study integrated system performance.
\end{abstract}

Prevalent throughout this ORM operation is a dynamically varying topology. In other words, the ORM starts with the SRMS grappled to the mated Shuttle/ISS stack (closed loop topology), moves to an open loop chain topology consisting of the Shuttle, SRMS, and ISS, and then, at the repair configuration, extends the chain topology to one consisting of the Shuttle, SRMS, ISS, and SSRMS/EVA crewman. The resulting long dynamic chain of vehicles and manipulators may exhibit significant motion between the Shuttle worksite and the EVA crewman due to the system flexibility throughout the topology (particularly within the SRMS/SSRMS joints and links). Since the attachment points of both manipulators span the flexible structure of the ISS, simulation analysis may also need to take that into consideration. Moreover, due to the lengthy time duration associated with the maneuver and repair, orbital effects become a factor and require the ISS vehicle control system to maintain active attitude control. 
Several facets of the ORM operation make the associated analytical efforts different from previous mission support, including: (1) the magnitude of the SRMS handled payload (i.e., Orbiter class), (2) the orbital effects induced on the integrated system consisting of the large Shuttle and ISS masses connected by a light flexible SRMS, (3) long duration environmental consequences due to the lengthy operational times associated with the maneuver and repair of the TPS, (4) active attitude control (as opposed to free drift) interacting with the SRMS and SSRMS manipulators (also due to the length of the maneuver and repair), (5) relative dynamics between the EVA crewman and the worksite influenced by the extended flexible topology.

In order to meet these analysis challenges, an ORM simulation architecture was developed leveraging upon numerous pre-existing simulation elements to analyze the various subsystems individually. For example, core manipulator subsystem simulations for both the SRMS and SSRMS were originally combined to provide the dual-arm dynamics topology simulation (in the absence of orbital dynamics and vehicle control). This capability was later merged with the simulation used to analyze SRMS loading with a heavy payload in the orbital environment with an active payload control system (in this case, the ISS Attitude Control System (ACS)), configured for the ORM: The resulting worksite dynamics simulation, based off of the modified ORM simulation, provided the extended topological chain of vehicles and manipulators, while taking into account the orbital effects of both the Shuttle and ISS (as well as its ACS).

Verification and validation (V\&V) of these integrated simulations became a challenge in itself. A systematic approach needed to be developed such that integration simulation results could be tested against previous constituent simulations upon which these simulations were built. General V\&V categories included: (1) core orbital state propagation, (2), stand-alone SRMS, (3) stand-alone SSRMS, (4) stand-alone ISS ACS, (5) integrated Shuttle, SRMS, ISS (with active ACS) in the orbital environment, and (5) dual-arm SRMS/SSRMS dynamics topology. Integrated simulation V\&V run suites were created and correlated to verification runs from subsystem simulations, in order to establish the validity of the results.

This paper discusses the simulation design challenges encountered while developing simulation capabilities to mirror the ORM operations. The paper also describes the incremental build approach that was utilized, starting with the subsystem simulation elements and integration into increasing more complex simulations until the resulting ORM worksite dynamics simulation had been assembled. Furthermore, the paper presents an overall integrated simulation V\&V methodology based upon a subsystem level testing, integrated comparisons, and phased checkout. 